\title{
"USING OF SOME PLANT EXTRACTS AND HONEYBEE PRODUCTS FOR FEEDING OF IMPORTED AND LOCAL HYBRIDS OF MULBERRY SILKWORM (Bombyx mori L.)" Safa Ismail ${ }^{1}$, S.H. Rateb ${ }^{2}$; K. Mohanna ${ }^{3}$ and M.H. Hussein ${ }^{4}$ 1,3- Plant Prot. Dept., Fac. of Agric., South Valley Univ.
} 2,4- Plant Prot. Dept., Fac. of Agric., Assiut Univ.

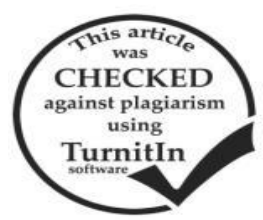

\section{ABSTRACT}

Nine aqueous or alcoholic plant extracts and three hive products were offered to $4^{\text {th }}$ and $5^{\text {th }}$ instar of imported and local hybrids of silkworm. They were: aqueous and alcoholic extracts of Eucalyptus, Mulberry, Christ's thorn (Hozaen and Baladi), Psidium, Ocimum basilicum, Mint, Budlia asiatica, Lantana camara, and Pollen, Bee Bread, Propolis. The measured or calculated parameters were: initial, final, average larval weight $(\mathrm{g})$ and growth index, silk glands weight and ratio \%, weight of cocoons, cocoon's shell and pupae, and cocoon shell ratio \%.

Concerning imported race of silkworm, feeding with mulberry leaves treated with aqueous extracts leads to significant increment of cocoon shell weight (g) in Eucalyptus, Christ's thorn (Baladi), O. basilicum, mint, pollen and propolis. Insignificant increase in cocoon shell ratio \% was noticed in Eucalyptus, Psidium, mint and propolis, while in case of alcoholic extracts, maximum significant shell weight was detected in Christ's thorn (Baladi) treatment, followed by: Eucalyptus, mulberry, Christ's thorn (Hozaen), Psidium, O. basilicum and mint, with significant differences with control. Insignificant increment in cocoon shell ratio \% was noticed in Psidium treatment only. All differences in treatments of imported race were significant with both of aqueous or alcoholic extracts, except cocoon shell ratio \%.

Concerning local race of silkworm, feeding with mulberry leaves which treated with tested aqueous extracts leads to significant increase in cocoon shell weight (gm) in Eucalyptus, mulberry, Christ's thorn (Hozaen), B. asiatica, L. camara, and pollen treatments, and insignificant increase in Christ's thorn (Baladi), Psidium, O. basilicum, and mint treatments. General increase in cocoon shell ratio \% in all treatments except bee bread and significant differences were detected in Eucalyptus and propolis treatments, while in case of alcoholic extracts significant increase in cocoon shell weight (gm) was found in B. asiatica treatment, followed by: eucalyptus, mulberry, Christ's thorn (Hozaen), L. camara, pollen and propolis treatments. Insignificant increment in cocoon shell ratio \% in propolis, L. camara, B. asiatica, mint and Eucalyptus. For local hybrid of silkworm, no significant differences in alcoholic treatments, were observed, however insignificant increment differences were detected in five treatments. Aqueous extracts of Eucalyptus or propolis increased cocoon shell ratio \% in both of tested hybrids. Aqueous or alcoholic extracts of B. asiatica or L. camara increased cocoon shell ratio \% in local hybrid. Aqueous extracts increased cocoon shell ratio \% in 15 cases, while in case of alcoholic extracts in 6 cases only.

The most effective treatments were: Eucalyptus, Psidium and propolis. Using of aqueous extracts was more effective available, and cheap as compared with using alcohol in extraction.

\section{INTRODUCTION}

The silkworms consume mulberry leaves in large quantities. The scope of the work of many researchers was observing their reaction to addition of various extracts for their food.

Rajeswari and Isaiarasu (2004) supplementation of Moringa olifera elicited varied responses in silkworm. Sridevi et al. (2004) used extracts of some medicinal plants, Terminalia arjuna, Withania somnifera, Tinospora cordifolia, and Leptedenia reticulata and found that all biological and economical parameters of silkworm were higher values compared with control. Murugesh and Baskar (2008) studied the effect of aqueous extracts of some plants on increasing of silk productivity of silkworm. Manjula et al. (2010) suggest that the administration of Dolichos lablab with mulberry leaves at $7.5 \%$ concentration was enhanced the biochemical reaction involved in the silk production.

Zah et al. (2001) reported that the olive oil is detrimental to the development of silkworm, regardless of the method of administration. Chandrakala et al. (2012) tested Parthenium hysterophorus extracts on young silkworm and concluded that utilizing of this extraction silkworm rearing is of special merits. Shahin et al. (2013) reported that lettuce seed oil, as a food additive, enhanced the productivity of silkworm. Karthikairaj et al. (2014) proved that herbal aqueous and alcoholic extracts of Ocimum, Acalypha, and Leucas can be exploited to control of microbial pathogens at the time of silkworm rearing and to get improved silk yield. Pardeshi and Bajad (2014a,b) used Amaranthus hybridus and Xanthium indicum extracts to improve the economic parameters of silkworm (Bombyx mori).

Aqueous leaf extract of Ocimum sanctum was tested by Sujatha et al. (2015). The same plant species was also tested by Padma et al. (2015) in India. Rateb and Abdel-Rahman (2015) tested some extracts including plant extracts on biological and economical parameters of silkworm.

This work aimed to study the effect of alcoholic and aqueous extracts of nine plant species and three honeybee hive products on the biological and economical characters of the silkworm (B. mori).

\section{MATERIALS AND METHODS}

This work was conducted in the Laboratory of Silkworm Rearing, Plant Protection Department, Faculty of Agriculture, Assiut University, during 2015.

Local and Bulgarian hybrid of silkworm were supplied from Sericulture Division, Plant Protection Institute, Ministry of Agriculture, Giza, to be used in the experimental work.

\section{I- Rearing of silkworm:}


The temperature during the incubation of silkworm eggs, was ranged from 23 to $25^{\circ} \mathrm{C}$, while the relative humidity was ranged from 85 to $90 \%$. Complete hatching took place, after few days of incubation. New leaves, or tender strips of mulberry leaves, were put over the hatching larvae, which crawled up the leaves, then these were removed with silkworms to the rearing place.

Normal method of silkworm rearing was carried out, till the beginning of $4^{\text {th }}$ instar, or beginning of tested treatments. The mulberry leaves for feeding of silkworm were cut early morning, then covered with wet clothes to protect than from loss of water. The leaves were cleaned and given to the first and second larval instars as strips or buds. Afterwards, the whole leaves was distributed in a usual manner four times/ day, till the beginning of fourth instar. Regular cleaning of rearing beds were carried out.

\section{II- Treatments:}

Nine alcoholic or aqueous plant extracts and three honeybee colony products were tested during this work. They were: Eucalyptus, Mulberry, Christ's thorn (Hozaen and Baladi), Psidium, Ocimum basilicum, Mint, Budlia asiatica, Lantana camara, and hive products: Pollen, Bee Bread and propolis. These extracts were sprayed on mulberry leaves and used for feeding of $4^{\text {th }}$ and $5^{\text {th }}$ instars larvae of imported and local hybrids silkworm (Bombyx mori).

For preparing of alcoholic or aqueous plant extracts: The dried leaves were extracted using $70 \%$ ethyl alcohol or dish water in a rate of $10 \mathrm{gms} / 100 \mathrm{ml}$ alcohol, in a water bath at $45^{\circ} \mathrm{C}$ for $48 \mathrm{hrs}$. Then filtered and the filtrated was completed to $100 \mathrm{ml}$ using alcohol $70 \%$.

All these treatments were used at concentration of $1 \%$ with water.

Every tested treatment was replicated three times in three carton boxes $(20.5 \times 19.5 \times 6.5 \mathrm{~cm})$, each contain fifty silkworms larvae and 150 larvae/treatment. Feeding with treated leaves was conducted four times/day. Control larvae were fed with untreated mulberry leaves. Twelve treatments were used during the work in addition to control.

\section{III- Criteria for evaluation:}

\section{1- Fresh weight of silkworms larvae:}

Twenty larvae were weight had initial and final weight, average weight and growth index were calculated 60 from each replicate were weighed (in gms), larvae/ treatment. Control larvae were also weighed. Total and mean weight/larvae (gm.) was calculated.

\section{2- Fresh weight of silk glands:}

Fifteen nine days old $5^{\text {th }}$ instar larvae, from each treatment were used for determination of silk glands weight (in gms). Control silk glands were also weighed (in gms). Total and mean weight (in gms) were calculated and ratio of glands weight body weight was calculated.

\section{3- Fresh weight of cocoons:}

From each replicate and treatments, good cocoons were collected, cut, opened, and pupae were weighed. The whole cocoon was weighed (in gms). Total and mean weight/cocoon (in gms), were calculated.

\section{4- Pupal weight:}

From each replicate and treatment, pupae were weighed (in gms). Total and mean weight/pupa (in gms) were estimated.

\section{5- Cocoon shell weight (C.S.W.):}

The previous cocoons were carefully opened and pupae were removed and cleaned from excuviae, then weighed. Total and mean weight/shell (in gms) were calculated.

6- Cocoon shell ratio (\%) (C.S.R.\%):

Cocoon shell ratio for each treatment was calculated as follows:

$$
\begin{aligned}
& \text { Cocoon shell ratio } \%=\frac{\text { Weight of cocoon shell }}{\text { Weight of cocoon }} \times 100 \\
& \text { Total and mean weight/shell (in gms.) were }
\end{aligned}
$$
calculated.

\section{7- Growth Index:}

Twenty five $5^{\text {th }}$ instar larvae of one, three, five and seven day old were weighed (in gms) and the following formula was used for calculation of the Growth Index:

$$
\text { Growth Index }=\frac{\text { Final weight of } 5^{\text {th }} \text { instar }- \text { Initial } 5^{\text {th }} \text { instar larval weight }}{\text { Initial larval weight of } 5^{\text {th }} \text { instar }(\mathrm{gms})}
$$

VI- Statistical analysis:

Obtained data were analysed using the factorial design. F-test was estimated for each analysis. The means were compared according to Duncan's Multiple Range Test.

\section{RESULTS AND DISCUSSION}

\section{I- Effects of used aqueous and alcoholic extracts on imported hybrid of silkworm summarized in Tables 1 and 2 , respectively.}

Table 1 show the effect of tested aqueous extracts on some growth parameters of silkworm. Maximum initial weight (gm) was detected n B. asiatica treatment, while maximum final weight (gm) and growth index of $5^{\text {th }}$ instar larvae was found in Christ's thorn treatment. Maximum and highly significant silk gland weight (gm) in Christ's thorn (Hozaen) treatment. General significant increment in average larval weight (gm) was noticed in all used treatments except in case of Psidium, L. camara, B. asiatica and bee bread, where this increment was insignificant.

Silk gland/larval weight ratio \% was generally decreased, except in mint treatment. Significant increase in fresh cocoon weight (gm) in Eucalyptus, and Christ's thorn treatments, while this increment was nonsignificant in mulberry, Psidium, O. basilicum, mint, L. camara and pollen treatments. Highly significant increase in pupal weight (gm) was measured in Christ's thorn (Baladi) treatment, only.

Signficiant increase in (C.S.W) in Eucalyptus, Christ's thorn (Baladi), mint, O. basilicum, pollen and propolis treatments. Insignificant increase in (C.S.R.\%) was detected in Eucalyptus, Psidium, mint, and propolis treatment. 
Effect of used alcoholic extracts on imported hybrid of silkworm is summarized in Table 2. Highest initial larval weight (gm) was detected in pollen treatment, while maximum final weight (gm) was found in Eucalyptus treatment, followed by: mulberry, Christ's thorn, Psidium, O. basilicum, mint, and bee bread treatments. Maximum larval growth index was found in mulberry treatment, followed by Eucalyptus, Christ's thorn, Psidium and $O$. basilicum, without significant differences.

Maximum significant increase in silk gland weight (gm) was detected in Christ's thorn (Baladi) treatment, followed with insignificant increment in Eucalyptus, mulberry, Christ's thorn (Hozaen), mint and $O$. basilicum trials. General significant increase in average larval weight (gm) except in $B$. asiatica, $L$. camara, bee bread and pollen treatments. General and significant decrease in silk gland ratio was calculated in Christ's thorn, $O$. basilicum, mint and pollen treatments.

Significant increment in fresh cocoon weight (gm) in Eucalyptus, mulberry Christ's thorn, mint and pollen trials, while maximum weight was in Christ's thorn (Baladi) treatment. Maximum and significant pupal weight $(\mathrm{gm})$ was in the last treatment, followed significantly by Christ's thorn (Hozaen), mulberry, mint, and pollen. Maximum significant figure of (C.S.W.) was measured in Christ's thorn (Bladi), followed by Eucalyptus, mulberry, Christ's thorn (Hozaen), Psidium, $O$. basilicum, and pollen treatments. Insignificant increase in (C.S.R.\%) was noticed in Psidium treatment.

Table 1: Effect of feeding imported silkworm on treated mulberry leaves with aqueous extracts.

\begin{tabular}{|c|c|c|c|c|c|c|c|c|c|c|c|c|c|c|c|c|c|c|c|}
\hline Parameters & 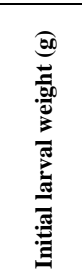 & 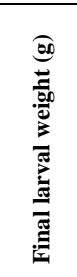 & Range & & $* *$ & 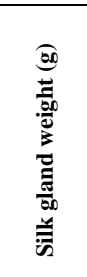 & $* *$ & 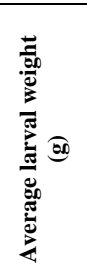 & $* *$ & 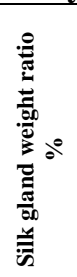 & $* *$ & 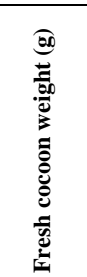 & $* *$ & 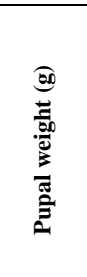 & $* *$ & 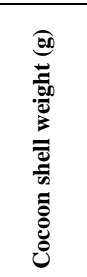 & $* *$ & 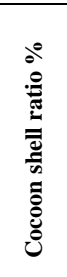 & NS \\
\hline 1- Eucalyptus & 0.6545 & 3.5310 & 2.8765 & 4.3950 & $\mathrm{BCDE}$ & 0.8415 & $\mathrm{AB}$ & 2.9726 & $\mathrm{BC}$ & 28.31 & $\mathrm{BC}$ & 1.4213 & $\mathrm{AB}$ & 1.0994 & $\mathrm{ABC}$ & 0.3219 & A & 22.65 & A \\
\hline 2- Mulberry & 0.6089 & 3.4868 & 2.8779 & 4.7264 & $\mathrm{AB}$ & 0.8096 & $\mathrm{ABC}$ & 3.2478 & $\mathrm{AB}$ & 24.93 & $\mathrm{CD}$ & 1.4044 & $\mathrm{ABCD}$ & 1.0998 & $\mathrm{ABC}$ & 0.3046 & B & 21.69 & $\mathrm{ABC}$ \\
\hline 3-Christsthom(Horaen) & 0.592 & 3.5459 & 2.9539 & 4.9897 & $\mathrm{AB}$ & 0.9110 & A & 3.2545 & $\mathrm{AB}$ & 27.99 & $\mathrm{BC}$ & 1.4145 & $\mathrm{ABC}$ & 1.1221 & $\mathrm{AB}$ & 0.2924 & $\mathrm{CD}$ & 20.67 & $\mathrm{BC}$ \\
\hline 4-Chiststhom(Baladi) & 0.618 & 3.7400 & 3.1220 & 5.0518 & $\mathrm{AB}$ & 0.8658 & $\mathrm{AB}$ & 3.2516 & $\mathrm{AB}$ & 26.63 & $\mathrm{BC}$ & 1.4696 & $\mathrm{~A}$ & 1.1609 & $\mathrm{~A}$ & 0.3087 & B & 21.00 & $\mathrm{ABC}$ \\
\hline 5- Psidium & 0.6046 & 3.4226 & 2.8180 & 4.6609 & $\mathrm{ABC}$ & 0.7825 & $\mathrm{BC}$ & 2.6829 & $\mathrm{CD}$ & 29.16 & $\mathrm{ABC}$ & 1.2954 & $\mathrm{CDEF}$ & 1.0137 & $\mathrm{BCD}$ & 0.2817 & $\mathrm{EF}$ & 21.75 & $\mathrm{ABC}$ \\
\hline 6-Ocinumbasilicum & 0.6209 & 3.3031 & 2.6822 & 4.3199 & $\mathrm{BCDE}$ & 0.8562 & $\mathrm{AB}$ & 3.3876 & $\mathrm{~A}$ & 25.27 & $\mathrm{CD}$ & 1.3986 & $\mathrm{ABCD}$ & 1.1019 & $\mathrm{AB}$ & 0.2967 & $\mathrm{C}$ & 21.22 & $\mathrm{ABC}$ \\
\hline 7- Mint. & 0.648 & 3.6119 & 2.9639 & 4.5739 & $\mathrm{ABCD}$ & 0.8579 & $\mathrm{AB}$ & 2.8645 & $\mathrm{BC}$ & 29.95 & $\mathrm{AB}$ & 1.3608 & $\mathrm{ABCDE}$ & 1.0665 & $\mathrm{ABCD}$ & 0.2943 & $\mathrm{C}$ & 21.62 & $\mathrm{ABC}$ \\
\hline 8-Budleia asiatica & 0.6601 & 2.7748 & 2.1147 & 3.2306 & $\mathrm{~F}$ & 0.7112 & $\mathrm{CD}$ & 2.5744 & $\mathrm{CD}$ & 27.63 & $\mathrm{BC}$ & 1.2239 & $\mathrm{~F}$ & 0.9631 & $\mathrm{D}$ & 0.2608 & $\mathrm{G}$ & 21.31 & $\mathrm{ABC}$ \\
\hline 9-Lantana camara & 0.6273 & 3.1551 & 2.5278 & 4.0297 & DE & 0.7058 & $\mathrm{CD}$ & 2.6382 & $\mathrm{CD}$ & 26.75 & $\mathrm{BC}$ & 1.3231 & $\mathrm{BCDEF}$ & 1.0616 & $\mathrm{ABCD}$ & 0.2615 & $\mathrm{G}$ & 19.76 & $\mathrm{C}$ \\
\hline 10- Pollen & 0.6403 & 3.3248 & 2.6845 & 4.1926 & $\mathrm{BCDE}$ & 0.7754 & $\mathrm{BC}$ & 2.8486 & $\mathrm{BC}$ & 27.22 & $\mathrm{BC}$ & 1.3429 & BCDEF & 1.0565 & $\mathrm{ABCD}$ & 0.2864 & DE & 21.33 & $\mathrm{ABC}$ \\
\hline 11- Bee Bread. & 0.643 & 3.1637 & 2.5207 & 3.9202 & E & 0.6939 & $\mathrm{CD}$ & 2.6356 & $\mathrm{CD}$ & 26.33 & $\mathrm{BC}$ & 1.2248 & $\mathrm{~F}$ & 0.9643 & $\mathrm{D}$ & 0.2604 & $\mathrm{G}$ & 21.26 & $\mathrm{ABC}$ \\
\hline 12- Propolis & 0.6266 & 3.0918 & 2.4652 & 3.9342 & E & 0.6387 & $\mathrm{D}$ & 2.9292 & $\mathrm{BC}$ & 21.80 & $\mathrm{D}$ & 1.2581 & $\mathrm{EF}$ & 0.9788 & $\mathrm{CD}$ & 0.2793 & $\mathrm{~F}$ & 22.20 & $\mathrm{AB}$ \\
\hline Control & 0.6305 & 3.2367 & 2.6062 & 4.1335 & $\mathrm{CDE}$ & 0.7616 & $\mathrm{BC}$ & 2.3379 & $\mathrm{D}$ & 32.58 & A & 1.2862 & DEF & 1.0072 & $\mathrm{BCD}$ & 0.2790 & $\mathrm{~F}$ & 21.69 & $\mathrm{ABC}$ \\
\hline $\begin{array}{ll}\text { LSD value at } & \\
0.05 & \text { alpha } \\
\text { level } & \\
0.01 & \\
\text { level } & \\
\end{array}$ & & & & & 0.5027 & & 0.1066 & & 0.3692 & & 3.7210 & & 0.1079 & & 0.1043 & 0.0062 & & & 1.617 \\
\hline
\end{tabular}

Table 2: Effect of feeding imported silkworm on treated mulberry leaves with alcoholic extracts.

\begin{tabular}{|c|c|c|c|c|c|c|c|c|c|c|c|c|c|c|c|c|c|c|c|}
\hline Treatments & 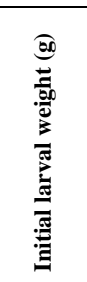 & 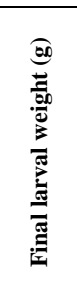 & Range & 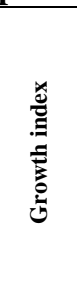 & $* *$ & 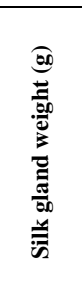 & $* *$ & 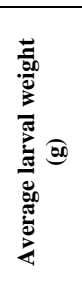 & $* *$ & 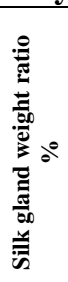 & $* *$ & 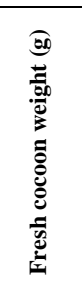 & $* *$ & 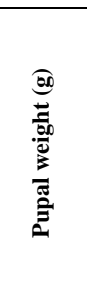 & $* *$ & 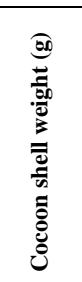 & $* *$ & 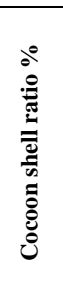 & NS \\
\hline 1- Eucalyptus & 0.6248 & 3.6370 & 3.0122 & .8211 & $\mathrm{AB}$ & 0.8669 & $\mathrm{AB}$ & 3.1047 & $\mathrm{AB}$ & 27.92 & $\mathrm{BCD}$ & 1.3926 & $\mathrm{ABC}$ & 1.0904 & $\mathrm{AB}$ & .3022 & $\mathrm{BC}$ & 21.70 & $\mathrm{AB}$ \\
\hline 2- Mulberry & 0.596 & 3.5477 & 2.9517 & 4.9525 & $\mathrm{AB}$ & 0.8797 & $\mathrm{AB}$ & 3.4264 & $A B$ & 25.67 & $\mathrm{CDE}$ & 1.4080 & $\mathrm{ABC}$ & 1.1102 & A & 0.2978 & $\mathrm{C}$ & 21.15 & $\mathrm{AB}$ \\
\hline 3-Chiststhom(Horaen) & 0.6229 & 3.4813 & 2.8584 & 4.5889 & $\mathrm{ABC}$ & 0.8896 & $\mathrm{AB}$ & 3.0654 & $\mathrm{ABC}$ & 29.02 & $\mathrm{ABCD}$ & 1.4396 & $\mathrm{AB}$ & 1.1377 & A & 0.3018 & $\mathrm{BC}$ & 20.97 & $\mathrm{AB}$ \\
\hline 4-Christsthom(Bahdi) & 0.6468 & 3.3698 & 2.7230 & 4.2100 & BCD & 0.9520 & A & 3.0278 & $\mathrm{ABC}$ & 31.44 & $\mathrm{ABCD}$ & 1.4571 & A & 1.1473 & A & 0.3098 & A & 21.26 & $\mathrm{AB}$ \\
\hline 5- Psidium & 0.6086 & 3.4416 & 2.8330 & 5.6549 & $\mathrm{ABC}$ & 0.7831 & $\mathrm{BC}$ & 2.8034 & $\mathrm{BCD}$ & 27.93 & $\mathrm{BCD}$ & 1.3356 & BCDE & 1.0350 & $\mathrm{ABC}$ & 0.3006 & $\mathrm{C}$ & 22.51 & A \\
\hline 6-Ocinumbasiliam & 0.6198 & 3.4740 & 2.8542 & 4.6047 & $\mathrm{ABC}$ & 0.8843 & $\mathrm{AB}$ & 3.0674 & $\mathrm{ABC}$ & 28.83 & $\mathrm{ABCD}$ & 1.3695 & $\mathrm{ABCD}$ & 1.0689 & $\mathrm{AB}$ & 0.3006 & $\mathrm{C}$ & 21.95 & $\mathrm{AB}$ \\
\hline 7- Mint. & 0.6285 & 3.1856 & 2.5571 & 4.0685 & $\mathrm{CDE}$ & 0.8029 & $\mathrm{BC}$ & 2.8882 & $\mathrm{BCD}$ & 27.80 & $\mathrm{BCD}$ & 1.4489 & $\mathrm{AB}$ & 1.1406 & A & 0.3083 & $\mathrm{AB}$ & 21.28 & $\mathrm{AB}$ \\
\hline 8-Budlearasiatica & 0.6529 & 2.9966 & 2.3437 & 3.5897 & $\mathrm{E}$ & 0.7236 & $\mathrm{C}$ & 2.6706 & $\mathrm{CDE}$ & 27.09 & BCDE & 1.3158 & $\mathrm{CDE}$ & 1.0461 & $\mathrm{AB}$ & 0.2696 & $\mathrm{E}$ & 20.49 & B \\
\hline 9Hantanacamara & 0.6516 & 2.9870 & 2.3354 & 3.5841 & $\mathrm{E}$ & 0.7227 & $\mathrm{C}$ & 2.4970 & $\mathrm{DE}$ & 28.94 & $\mathrm{ABCD}$ & 1.2540 & $\mathrm{EF}$ & 0.9869 & $\mathrm{BC}$ & 0.2671 & $\mathrm{E}$ & 21.30 & $\mathrm{AB}$ \\
\hline 10- Pollen & 0.6574 & 3.2340 & 2.5766 & 3.9194 & DE & 0.7878 & $\mathrm{BC}$ & 2.6723 & $\mathrm{CDE}$ & 29.48 & $\mathrm{ABC}$ & 1.4206 & $\mathrm{ABC}$ & 1.1242 & A & 0.2963 & $\mathrm{C}$ & 20.86 & $\mathrm{AB}$ \\
\hline 11- Bee Bread. & 0.6505 & 2.9801 & 2.3296 & 3.5810 & $\mathrm{E}$ & 0.5639 & D & 2.5503 & $\mathrm{DE}$ & 22.11 & $\mathrm{E}$ & 1.2476 & $\mathrm{EF}$ & 0.9957 & $\mathrm{BC}$ & 0.2519 & G & 20.19 & B \\
\hline 12- Propolis & 0.6363 & 3.1578 & 2.5215 & 3.9628 & $\mathrm{DE}$ & 0.6892 & $\mathrm{C}$ & 2.8811 & $\mathrm{BCD}$ & 23.92 & DE & 1.1895 & $\mathrm{~F}$ & 0.9288 & $\mathrm{C}$ & 0.2607 & $\mathrm{~F}$ & 21.91 & $\mathrm{AB}$ \\
\hline Control & 0.6033 & 3.2162 & 2.6129 & 4.3310 & $\mathrm{ABCD}$ & 0.7947 & $\mathrm{BC}$ & 2.3464 & E & 33.87 & A & 1.2781 & DEF & 0.9966 & $\mathrm{BC}$ & 0.2815 & D & 22.03 & A \\
\hline $\begin{array}{l}\text { LSD value at } \\
0.05 \text { alpha } \\
\text { level } \\
0.01 \text { alpha } \\
\text { level }\end{array}$ & & & & & 0.5538 & & 0.119 & & 0.3653 & & 4.704 & & 0.1005 & 0.09 & & 0.006 & & & 1.5 \\
\hline
\end{tabular}

Thus it is possible to say that, in imported hybrid of silkworm all differences between tested treatments 
were highly significant in case of aqueous or alcoholic extracts, except in (C.S.R.\%), where it was insignificant with both of aqueous or alcoholic extracts.

Effect of plant extracts was studied in India by Jeyapaul et al. (2003). Eswaran and Savarkodiyone (2004) used 1\% aqueous extract tapioca flour and Amaranthus leaves for feeding of silkworms.

II- Effects of used aqueous and alcoholic extracts on local hybrid of silkworm summarized in Tables 3 and 4 , respectively.

Effect of examined aqueous extracts on local hybrid of silkworm is summarized in Table 3 . Maximum initial larval weight $(\mathrm{gm})$ was found in $O$. basilicum treatment. General increase in silk gland weight (gm), except with bee bread was detected. It was significant in Christ's thorn (Hozaen) followed by: Christ's thorn, Psidium, mint and L. camara. A general increase in silk gland ratio was detected. It was significant in Psidium, followed by: Eucalyptus, Christ's thorn, O. basilicum, mint, L. camara, and pollen treatments.

Insignificant increment in cocoon fresh weight
L. camara, bee bread and maximum figure was notied in pollen treatment. Insignificant increase in pupal weight $(\mathrm{g})$ was measured in: mulberry, followed by: Christ's thorn, B. asiatica, L. camara, pollen and bee bread treatments.

General increase in (C.S.W) in all treatments, except bee bread. It was significant in: Eucalyptus, mulberry, Christ's thorn (Hozaen), $P$. asiatica, $L$. camara, and pollen treatments. A general increment in (C.S.R.\%) was noticed in all examined aqueous extracts, except in bee bread treatments. This increment was significant in Eucalyptus and propolis trials.

Rajeswari and Isaiarasu (2004) found that extracts of Moringa oliefera (1\% w/v) elicited a responses in the final instar larvae. The same was noticed in our work.

Hiware and Bhalerao (2008) used aqueous extracts of two medicinal plants and found interesting and pesticide results with respect to many parameters.

Effect of used alcoholic extracts on local hybrid of silkworm are summarized in Table 4. Maximum initial weight (gm) was found in $O$. basilicum treatment. (gm) was found in Eucalyptus, mulberry, Christ's thorn,

Table 3: Effect of feeding local silkworm on treated mulberry leaves with aqueous extracts.

\begin{tabular}{|c|c|c|c|c|c|c|c|c|c|c|c|c|c|c|c|c|c|c|c|}
\hline Treatments & 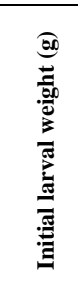 & 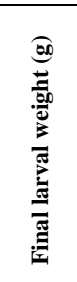 & Range & 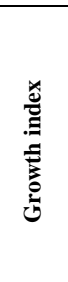 & $* *$ & 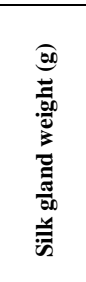 & NS & 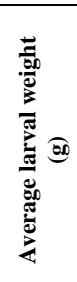 & $* *$ & 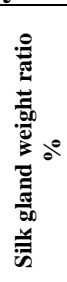 & ** & 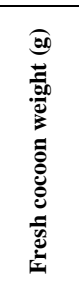 & ** & 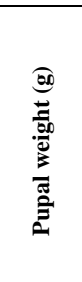 & $* *$ & 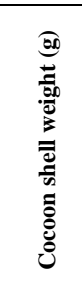 & ** & 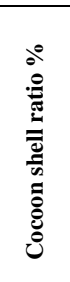 & ** \\
\hline $1-E u$ & 660 & 2.828 & 2.1 & 3.2814 & $\mathrm{ABCl}$ & 0.8324 & $\mathrm{AB}$ & 2.5663 & $\mathrm{CDE}$ & 32.44 & $\mathrm{AB}$ & 1.1606 & $\mathrm{ABC}$ & 0.9095 & $\mathrm{ABC}$ & 0.2511 & $\mathrm{AB}$ & 21.64 & $\mathrm{AB}$ \\
\hline $2-\mathrm{Mu}$ & 7556 & 2.9390 & 2.1834 & 2.8896 & $\mathrm{DE}$ & 0.7649 & $\mathrm{ABC}$ & 2.6947 & $\mathrm{ABCDE}$ & 28.01 & BCD & 1.2366 & $\mathrm{ABC}$ & 0.9863 & A & 0.2503 & $\mathrm{AB}$ & 20.24 & $\mathrm{BC}$ \\
\hline 3Chisth & 6968 & 3.0265 & 2.3297 & 3.3434 & $\mathrm{ABCD}$ & 0.8983 & A & 2.8402 & $\mathrm{ABC}$ & 31.63 & $\mathrm{AB}$ & 1.1887 & $\mathrm{ABC}$ & 0.9354 & $\mathrm{ABCD}$ & 0.2534 & A & 21.32 & $\mathrm{ABC}$ \\
\hline 4Chiststhom(Baladi) & 6974 & 2.8605 & 2.1631 & 3.1017 & BCDE & 0.8518 & A & 2.5915 & $\mathrm{BCDE}$ & 32.87 & $\mathrm{AB}$ & 1.1514 & $\mathrm{ABCD}$ & 0.9159 & $\mathrm{ABCD}$ & 0.2355 & $\mathrm{ABC}$ & 20.45 & $\mathrm{ABC}$ \\
\hline 5- Psidium & 0.6299 & 2.8710 & 2.2411 & 3.5579 & $\mathrm{AB}$ & 0.8575 & A & 2.4739 & $\mathrm{DE}$ & 34.66 & A & 1.0824 & $\mathrm{CD}$ & 0.8533 & $\mathrm{CD}$ & 0.2291 & $\mathrm{BC}$ & 21.17 & $\mathrm{ABC}$ \\
\hline 6-Ocinumbasilicum & 0.8197 & 2.7514 & 1.9317 & 2.3566 & $\mathrm{~F}$ & 0.8071 & $\mathrm{AB}$ & 2.7711 & $\mathrm{ABCDE}$ & 29.13 & $\mathrm{ABC}$ & 1.1196 & $\mathrm{BCD}$ & 0.8809 & $\mathrm{BCD}$ & 0.2387 & $\mathrm{ABC}$ & 21.32 & $\mathrm{ABC}$ \\
\hline 7- Mint. & 0.609 & 2.7037 & 2.0947 & 3.4396 & $\mathrm{ABC}$ & 0.8632 & A & 2.5720 & $\mathrm{CDE}$ & 33.56 & $\mathrm{AB}$ & 1.0587 & D & 0.8337 & D & 0.2250 & $\mathrm{C}$ & 21.25 & $\mathrm{ABC}$ \\
\hline 8-Budleia a & 0.759 & 2.8762 & 2.1172 & 2.7895 & $\mathrm{EF}$ & 0.8230 & $\mathrm{AB}$ & 2.9048 & $\mathrm{ABC}$ & 28.33 & BCD & 1.2265 & A & 0.9723 & $\mathrm{AB}$ & 0.2541 & A & 20.72 & $\mathrm{ABC}$ \\
\hline 9-Lantana camara & 0.7875 & 2.9577 & 2.1702 & 2.7558 & $\mathrm{EF}$ & 0.8542 & A & 2.9173 & $\mathrm{AB}$ & 29.28 & $\mathrm{ABC}$ & 1.1833 & $\mathrm{AB}$ & 0.9338 & $\mathrm{ABCD}$ & 0.2495 & $\mathrm{AB}$ & 21.09 & $\mathrm{ABC}$ \\
\hline 10- Pollen & 6858 & 2.7362 & 2.0504 & 2.9898 & $\mathrm{CDE}$ & 0.8221 & $\mathrm{AB}$ & 2.4538 & $\mathrm{DE}$ & 33.50 & $\mathrm{AB}$ & 1.2268 & A & 0.9783 & A & 0.2485 & $\mathrm{AB}$ & 20.26 & $\mathrm{BC}$ \\
\hline Bread. & 0.6394 & 2.7307 & 2.0913 & 3.2707 & $\mathrm{ABCDE}$ & 0.6151 & $\mathrm{C}$ & 2.3961 & E & 25.67 & $\mathrm{CD}$ & 1.1483 & $\mathrm{ABCD}$ & 0.9654 & $\mathrm{AB}$ & 0.1829 & $\mathrm{D}$ & 15.93 & D \\
\hline polis & 0.7369 & 2.8328 & 2.0 & 2.8442 & DEF & 0.7557 & $\mathrm{ABC}$ & 2.6586 & $\mathrm{ABCDE}$ & 28.42 & $\mathrm{BCD}$ & 1.1075 & $\mathrm{BCD}$ & 0.8620 & $\mathrm{CD}$ & 0.2455 & $\mathrm{ABC}$ & 22.17 & A \\
\hline Control & 0.6865 & 3.1938 & 2.5073 & 3.6523 & A & 0.6772 & BC & 2.9391 & A & 23.04 & D & 1.1390 & $\mathrm{ABCD}$ & 0.9148 & $\mathrm{ABCD}$ & 0.2243 & C & 19.69 & C \\
\hline $\begin{array}{l}\text { LSD value at } \\
0.05 \text { alpha level } \\
0.01 \text { alpha level }\end{array}$ & & & & & 0.4746 & & 0.1410 & & 0.2967 & & 5.0610 & & 0.0859 & & 0.0812 & & 0.0197 & & 1.5940 \\
\hline
\end{tabular}

Table 4: Effect of feeding of local silkworm on treated mulberry leaves with alcoholic extracts.

\begin{tabular}{|c|c|c|c|c|c|c|c|c|c|c|c|c|c|c|c|c|c|c|c|}
\hline \multicolumn{20}{|l|}{ Parameters } \\
\hline I atameters & 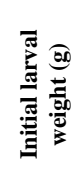 & 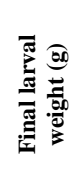 & Range & 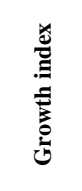 & $* *$ & 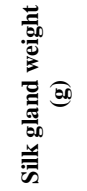 & $* *$ & 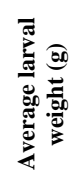 & $* *$ & 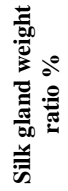 & $* *$ & 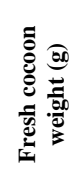 & $* *$ & 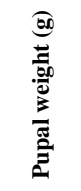 & $* *$ & 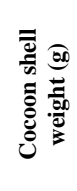 & $* *$ & 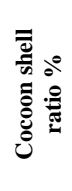 & $* *$ \\
\hline \multicolumn{20}{|l|}{ Treatments } \\
\hline 2- Mulberry & 0.7105 & 2.9472 & 2.2367 & 3.1481 & $\mathrm{BCD}$ & 0.7602 & $\mathrm{BC}$ & 2.6961 & $\mathrm{AB}$ & 28.20 & $\mathrm{CDE}$ & 1.2197 & $\mathrm{~A}$ & 0.9751 & A & 0.2446 & $\mathrm{AB}$ & 20.05 & A \\
\hline 3-Chistshom(Hozaen) & 0.6948 & 2.8954 & 2.2006 & 3.1672 & $\mathrm{BCD}$ & 0.8445 & $\mathrm{ABC}$ & 2.5141 & B & 33.59 & $\mathrm{BC}$ & 1.2287 & A & 0.9800 & A & 0.2487 & A & 20.24 & A \\
\hline 4-Chiststhom (Baladi) & 0.7506 & 3.0353 & 2.2847 & 3.0438 & $\mathrm{BCD}$ & 0.8745 & $\mathrm{AB}$ & 2.8317 & $\mathrm{AB}$ & 30.88 & $\mathrm{BCD}$ & 1.1825 & A & 0.9422 & A & 0.2403 & $\mathrm{ABCD}$ & 20.32 & A \\
\hline 5-Psidium & 0.6589 & 2.6892 & 2.0303 & 3.0813 & BCD & 0.9400 & A & 2.4139 & B & 38.94 & A & 1.0675 & $\mathrm{BC}$ & 0.8419 & $\mathrm{BC}$ & 0.2256 & $\mathrm{BCDE}$ & 21.13 & A \\
\hline 6-Ocinumbasiliaum & 0.8345 & 3.0346 & 2.2001 & 2.6364 & DE & 0.7367 & $\mathrm{CD}$ & 2.5344 & B & 29.07 & $\mathrm{CDE}$ & 1.1765 & A & 0.9332 & A & 0.2433 & $\mathrm{ABCD}$ & 20.68 & A \\
\hline 7-Mint & 0.6231 & 2.9189 & 2.2958 & 3.6845 & A & 0.7767 & $\mathrm{BC}$ & 2.8009 & $\mathrm{AB}$ & 27.73 & $\mathrm{DE}$ & 1.0286 & $\mathrm{C}$ & 0.8080 & $\mathrm{C}$ & 0.2206 & DE & 21.45 & A \\
\hline 8-Budleiaasiatica & 0.7601 & 3.1842 & 2.4241 & 3.1892 & $\mathrm{BC}$ & 0.8694 & $\mathrm{ABC}$ & 3.0867 & A & 28.17 & DE & 1.2463 & A & 0.9855 & A & 0.2608 & A & 20.93 & A \\
\hline 9-Lantamacamara & 0.8019 & 2.6653 & 1.8634 & 2.3237 & E & 0.8829 & $\mathrm{AB}$ & 2.5133 & B & 35.13 & $\mathrm{AB}$ & 1.1813 & A & 0.9317 & A & 0.2496 & A & 21.13 & A \\
\hline 10- Pollen & 0.7079 & 2.7814 & 2.0735 & 2.9291 & BCD & 0.8201 & $\mathrm{ABC}$ & 2.6518 & B & 30.93 & $\mathrm{BCD}$ & 1.2255 & A & 0.9748 & A & 0.2507 & A & 20.46 & A \\
\hline Bread. & 0.6328 & 2.7599 & 2.1271 & 3.3614 & $\mathrm{AB}$ & 0.5793 & $\mathrm{E}$ & 2.0392 & $\mathrm{C}$ & 28.41 & $\mathrm{CDE}$ & 1.1500 & $\mathrm{AB}$ & 0.9450 & A & 0.2050 & $\mathrm{E}$ & 17.83 & B \\
\hline polis & 0.7416 & 2.7820 & 2.0404 & 2.7513 & $\mathrm{CDE}$ & 0.7673 & $\mathrm{BC}$ & 2.5949 & B & 29.57 & BCD & 1.1656 & A & 0.9140 & $\mathrm{AB}$ & 0.2516 & A & 21.59 & A \\
\hline Control & 0.67 & 3.2106 & 2.5406 & 3.7919 & A & 0.6313 & $\mathrm{DE}$ & 2.6753 & B & 23.60 & E & 1.0619 & BC & 0.8397 & BC & 0.2222 & $\mathrm{CDE}$ & 20.92 & A \\
\hline $\begin{array}{l}\text { LSD value at } \\
0.05 \text { alpha } \\
\text { level } \\
0.01 \text { alpha }\end{array}$ & & & & & 0.4646 & & 0.1192 & & 0.3614 & & 4.8450 & & 0.0859 & & 0.0812 & & 0.0197 & & 1.6670 \\
\hline
\end{tabular}


Maximum and significant increase in mean larval. Weight was detected in B. asiatica treatment, followed by insignificant increase in mulberry, Christ's thorn (Baladi) and mint. General increment in silk gland ratio was noticed. It was significant in Eucalyptus, Christ's thorn (Hozaen), Psidium, B. asiatica, L. camara and proplis.

Concerning fresh cocoon weight $(\mathrm{g})$ : significant increase was found in $B$. asiatica, followed by mulberry, Christ's thorn, A. basilicum, pollen, $L$. camara, and propolis.

Significant increase in pupal weight (gm) was noticed in treatments Christ's thorn (Hozaen), followed by mulberry, Christ's thorn (Baladi), O. basilicum, $B$. asiatica, L. camara, pollen and bee bread. Significant increment in (C.S.W.) was noticed in $B$. asiatica, followed by: eucalyptus, mulberry, Christ's thorn (Hozaen), L. camara, pollen and propolis. Insignificant increment, was noticed in (C.S.R.\%), when alcoholic extracts of propolis, L. camara, B. asiatica, mint and Eucalyptus, were used.

Concerning feeding local hybrid of silkworm, on mulberry leaves which sprayed with alcoholic extracts, insignificant increment in (C.S.R.\%) in five treatments.

From the above mentioned results, it can be concluded that aqueous extracts of Eucalyptus and propolis leads to increment in (C.S.R.\%), in both of imported or local hybrids of silkworm. Aqueous or alcoholic extracts, increased (C.S.R.\%) in local hybrid. Aqueous extracts increase (C.S.R.\%) in 15 cases, while with alcoholic extracts it was detected in 6 cases only. This reflects the merits of using water extraction, and in the same time it is more cheap and available, as compared with using alcoholic extraction.

The best and more effective treatments during this work were: Eucalyptus, Psidium, and propolis.

\section{REFERENCES}

Chandrakala, M.V.; P. Dharani; R.R. Patil; J. Sukumar; V.G. Maribashetty; C.S. Gururaj; C. Shivakumar and B.M. Sekharappa (2012): Investigation on the application of Parthenium hysterophorus extracts as feed additives for young larvae of silkworm, Bombyx mori L. Agric. Sci. Res. J. 2(8): 449-452.

Eswaran, R. and S. Sevarkodiyone (2004): Comparative effect of tapioca flour and Amaranthus green leaves on feeding characters of mulberry silkworm, Bombyx mori L. Bionotes. 6 (3): 88-89.

Hiware, C.J. and R.S. Bhalerao (2008): Effect of some medicinal plant extracts on the economic characters of mulberry silkworm, Bombyx mori L. Flora and Fauna (Jhansi), 14 (1): 90-92.

Jeyapaul, C.; C. Padmalatha; A.J.A. R Singh; A.G. Murugesan and P. Dhasarathan (2003): Effect of plant extracts on nutritional efficiency in mulberry silkworm, Bombyx mori L. Indian J. Seric., 42 (2): 128-131.
Karthikairaj, K.; L. Isaiarasu and N. Sakthivel (2014): Efficacy of some herbal extracts on microbes causing flacherie disease in mulberry silkworm, Bombyx mori L. J. Biopest 7: 89-93.

Manjula, S.; S. Selvi; M. Veeranarayanan and S. Nadanam (2010): Biochemical alterations in the haemolymph of silkworm Bombyx mori (L.) (Lepidoptera: Bombycidae) fed with mulberry leaves enriched with Indian bean (Dolichos lablab). Recent Res. Sci. \& Technol., 2 (3): 32-37.

Murugesh, K.A. and R.N. Bhaskar (2008): Eco-friendly approach to increase silk productivity of mulberry silkworm, Bombyx mori L. Mysore J. Agric. Sci., 42 (4): 678-683.

Padma, S.V.; P. Devi and B.M. Ramani (2015): Effect of Ocimum sanctum L. plant extract on the economic parameters of silkworm, Bombyx mori L. J. of Entomology and Zoology Studies, 3 (2): 62-64.

Pardeshi, A.B. and P.N. Bajad (2014a): The effect of nutritional supplementation with Amaranthus hybridus Linn. extract on economic performance of mulberry silkworm, Bombyx mori L. Sch. Acad. J. Biosci., 2 (4): 272-276.

Pardeshi, A.B. and P.N. Bajad (2014b): Effect of Xanthium indicum Linn. plant extract on the economic parameters of silkworm, Bombyx mori L. International J. of Recent Scientific Res., 5 (3): 683-686.

Rajeswari, K. and L. Isaiarasu (2004): Influence of the leaf, flower and pod extracts of Moringa oleifera on the growth and reproductive parameters of Bombyx mori L. J. Entomol., 29 (4): 331-338.

Rateb, S.H. and Y.A. Abdel-Rahman (2015): Effects of some extracts on growth characters of mulberry silkworm (Bombyx mori L.). Assiut J. of Agric. Sci. (In Press).

Shahin, R.; G. Gadelhak; S.M. Mahmoud; E.I. ElAgamy and M. Idriss (2013): The effect of lettuce seed oil on some economical parameters of the mulberry silkworm Bombyx mori. J. of Applied Sci. Res., 9 (4): 2702-2707.

Sridevi, G.; R.N. Bhaskar; M.C Devaiah; R. Govindan; M. Vasundhara and K.R. Geetha (2004): Effect of medicinal plant extracts on rearing parameters of silkworm, Bombyx mori L. (PM x CSR2). Bull. Indian Acad. Seric.,8(2): 22-29.

Sujatha, K.; J. Sathish, and J. Anitha (2015): Effect of medicinal botanical(Ocimum sanctum), family, labiateae on commercial parameters of the silkworm,Bombyx.mori,L.Int. J. of Multidisciplinary and Current Research,3:76-78.

Zah, C.; L.A. Mărghitas; A. Mater and M.N. Mădas (2011): The effect of dietary supplements on the development of Bombyx mori L. silkworm. Animal Sci. and Biotechnologies, 44 (1): 153157. 
استخدام بعض المستخلصـات النباتيـة ومنتجـات طائفة النحل الهجين المستورد والمحلي لدودة الحريـر التوتيـة

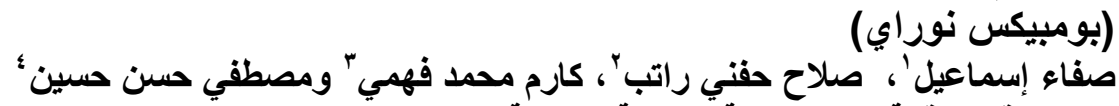

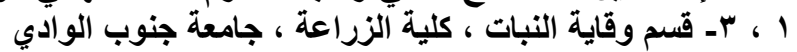

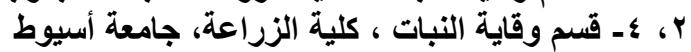

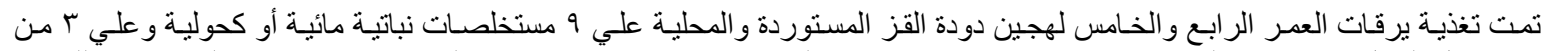

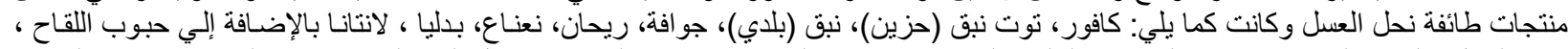

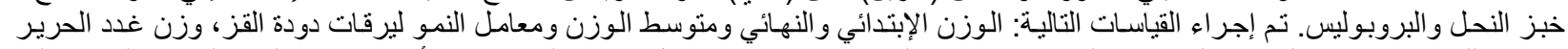

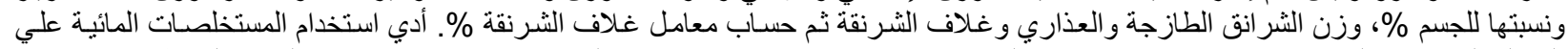

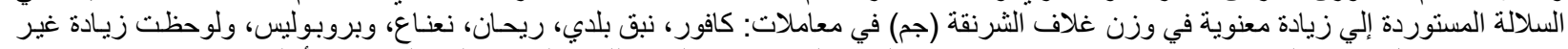

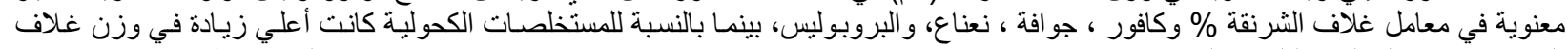

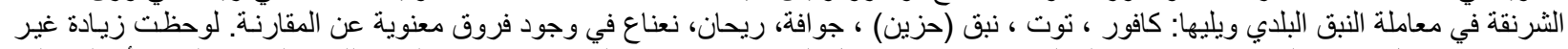

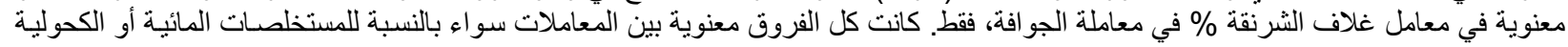

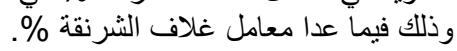

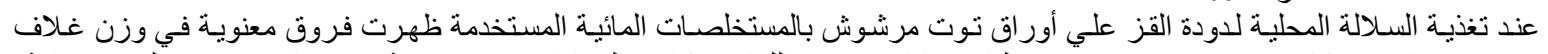

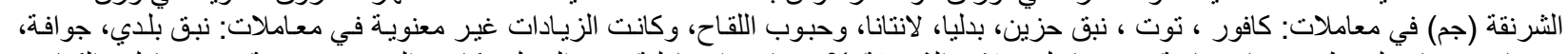

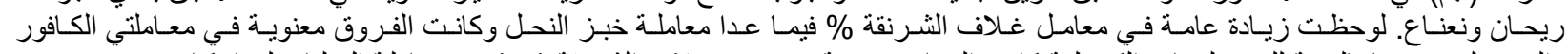

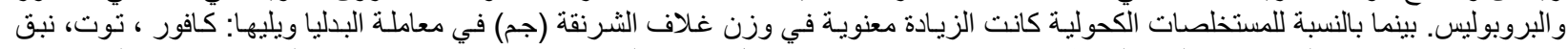

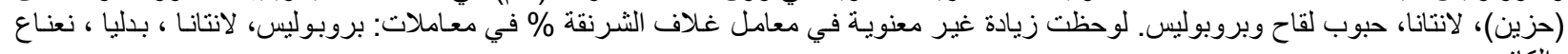

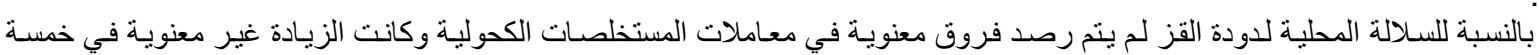

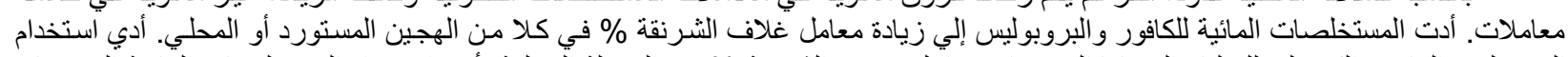

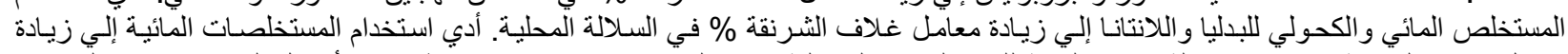

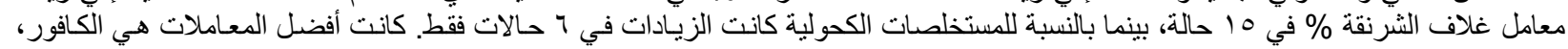

إن استخدام الماء في عملية الاستخلاص أرخص و أفضل كثير أ من استخدام الكحول في هذا الشأن.

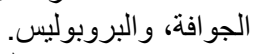

